\title{
Using the fractal dimension method to assess ossification after open sinus lift surgery
}

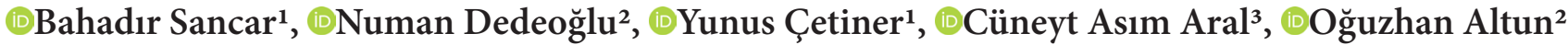 \\ ${ }^{1}$ İnönü University, Faculty of Dentistry, Department of Oral and Maxillofacial Surgery, Malatya, Turkey \\ 2İnönü University, Faculty of Dentistry, Department of Oral and Maxillofacial Radiology, Malatya, Turkey \\ ${ }^{3}$ İnönü University, Faculty of Dentistry, Department of Periodontology, Malatya, Turkey
}

Cite this article as: Sancar B, Dedeoğlu N, Çetiner Y, Aral CA, Altun O. Using the fractal dimension method to assess ossification after open sinus lift surgery. J Health Sci Med 2022; 5(1): 326-330.

\begin{abstract}
Objective: The present study aims to use the fractal dimension method to assess ossification occurring in patients undergoing an open sinus lift surgery performed with the use of xenograft.

Material and Method: In our study, we used 90 orthopantomographs of a total of 43 patients. Our study consists of three groups: Group A, Group B, and Group C. Using the fractal dimension method, we assessed the orthopantomographs taken within three to six months after the open sinus lift surgery (Group A), taken after six to nine months after the open sinus lift surgery (Group B), and taken more than nine months after the open sinus lift surgery (Group C). The data were analyzed using IBM SPSS V23. The compliance of the data with the normal distribution was examined using the Shapiro-Wilk test.

Result: The three-way statistics made between the mean values of the groups revealed a difference $(p=0,033)$. The density of the xenograft material in the study area tended to decrease starting from the period of three to six months after the surgery.

Conclusion: The fractal dimension method can be used to assess ossification occurring after open sinus lift surgery that is performed with the use of xenografts.
\end{abstract}

Keywords: Fractal dimension/open sinus lift/xenograft

\section{INTRODUCTION}

Dental implant treatment is an effective option for the replacement of lost teeth and for the retention and stability of removable dentures and maxillofacial prostheses. The alveolar bones of the jaws undergo continuous resorption after tooth loss (1). After the extraction of teeth, the alveolar resorption in the posterior maxilla and the increasing pneumatization of the maxillary sinus limit the amount and quality of bone needed for implant placement in this region $(2,3)$. Although the usage of short or curved implants has been indicated as a viable therapeutic option to overcome these obstacles (4), sinus grafting emerges as a good option to facilitate implant placement, considering the anatomical constraints and the long-term biomechanical stability of the prosthesis (3). Therefore, a variety of methods have been needed to obtain the necessary bone structure in the sinus region. Among the sinus augmentation procedures, the techniques of the lateral window and sinus floor elevation (performed with an osteotome) have gained recognition in treating the loss of vertical bone height in the posterior maxilla (1).
Bone grafting material is one of the factors that play an important role in sinus grafting outcomes. Xenografts are biocompatible materials that offer a structure and physical characteristics that are similar to human cancellous bone (3). The inorganic bovine bone combined with autogenous bone can be a perfect graft material for maxillary sinus augmentation (5). This can be explained by the slow absorption rate and osteoconductive characteristics of the inorganic xenograft (6).

Many methods have been put forward to examine trabecular structures, however, the most commonly used one today is the fractal dimension (FD) method, and it is indicated with the value of fractal dimension (FD) in numerical terms (7). The word fractal derives from the Latin word fractus, which means fractured (8). For a long time, fractal geometry has been used to identify irregular patterns which exhibit self-similarity at different scales (7). Fractals are especially used in the characterization of a porous medium (9). Again, the fractal analysis method is widely used for image analysis and pattern 
recognition (7), and the spongiform bone exhibits fractal characteristics due to its reticulated structure. Therefore, fractal geometry and fractal dimension measurements can be used to identify the complex structure of trabecular bone (10). The magnified image which monitors the internal porous structure of the bone at different scales resembles the initial original unmagnified appearance (7). Generally, as the size scale increases, the shape gets equally complex (11). It is specified that the FD obtained from orthopantomography indicates the changes in the density of the spongiform bone and the mineral loss in the bone (12).

Bone tissue consists of two parts, cortical and cancellous, and the ratio of these two structures in the entire bone is approximately $80 \%$ and $20 \%$, respectively (13). Due to its proximity to the bone marrow cavity, remodeling is more apparent in cancellous bone, which is the most metabolically active component of the skeleton (14). However, cancellous bone is also extremely susceptible to disruptions induced by local or systemic factors that can cause a significant imbalance in bone turnover (14).

In our study, we aimed to assess the grafted area with a fractal analysis of orthopantomographs taken during the postoperative follow-up of the patients who were treated with open sinus lift surgery. This study hypothesizes that fractal analysis can be used to assess ossification occurring in the operated area after open sinus lift operations performed with the use of xenograft. We could not find any other study in the literature using fractal analysis to assess, based on the postoperative periods, the area undergoing open sinus lift surgery.

\section{MATERIAL AND METHOD}

This study was carried out at İnönü University Faculty of Dentistry, Department of Oral, Dental, and Maxillofacial Radiology. All procedures were carried out in accordance with the ethical rules and the principles of the Declaration of Helsinki. In this study, images obtained by using planmeca proline XC (2009, 60-80 kVP, 4-12 $\mathrm{mA}, 18$ second exposure time, Helsinki, Finland) panoramic device were used. In our study, we used 90 orthopantomographs of a total of 43 patients who were treated with open sinus lift surgery between September 2018 and June 2021. Fractal analysis was performed on the orthopantomographs taken three to six months after the surgery (A), six to nine months after the surgery (B), and more than nine months after the surgery $(\mathrm{C})$. The study included patients who were treated with bilateral or unilateral open sinus lift surgery performed with the use of xenograft (Apatos, Osteobiol). The study excluded the patients with systemic problems that would endanger the bone healing process, the patients with severe parafunctional habits, the patients with substance abuse, and the patients with severe periodontal disease. Care was taken not to let any anatomic structures, such as tooth roots, be visible in the images obtained from panoramic radiographs. After determining an area of 20x20 pixels using the most superior region of the graft placed, fractal dimension (FD) analysis was performed in the maxilla using the box-counting method. The operations related to fractal dimension analysis were carried out by the same person on the same computer. Fractal dimension analysis was performed in the ImageJ image analysis software, which is a version of the National Institute of Health Image, using the method developed by White and Rudolph. (15). Fractal dimension analysis operations were performed in the following order: After the relevant area to be examined in the image was cropped, it was saved and copied in the 8-bit format. Then, a Gaussian filter (sigma $=35$ pixels) was applied to the duplicated image, and the image was blurred. The image (which was blurred by applying the Gaussian filter) was then subtracted from the original image by the 'subtraction' process. A brightness value of 128 was added to each pixel location. Regardless of the initial brightness of the image, 128 was determined as the threshold value. The image taken as the threshold value was converted to binary format. To avoid redundancy in the image, erosion and dilatation processes were applied to the image. The inverted image was skeletonized, and in this way, it was made sure that only the central parts of the trabeculae remained. In the ImageJ software, FB analysis was performed on the skeletonized image by using the 'box-counting' function.

\section{Statistical Method}

The data were analyzed using IBM SPSS V23. The compliance of the data to normal distribution was examined using the Shapiro-Wilk test. To compare the intragroup values that exhibited normal distribution according to gender, the independent two-sample t-test was used. To compare the data that exhibited normal distribution according to groups, the one-way analysis of variance (ANOVA) was used. Also, multiple comparisons were examined with the Tukey HSD test. For quantitative data, the analysis results were presented as mean \pm standard deviation and median (minimummaximum). The level of significance was taken as $\mathrm{p}<0.05$.

\section{RESULTS}

A significant difference was found between the mean values of the groups $(p=0.033)$. The mean value was 0.97 in Group A, 0.96 in Group B, and 0.92 in Group C. While the mean value in Group B did not exhibit any difference compared to Groups A and C, the mean value of Group A was higher than that of Group C (Table 1). 


\begin{tabular}{|ccccc|}
\hline \multicolumn{4}{|c|}{ Table 1. Comparison of the values according to groups } \\
\hline Groups & $\begin{array}{c}\text { Number of } \\
\text { samples }\end{array}$ & $\begin{array}{c}\text { Mean } \pm \text { S. } \\
\text { deviation }\end{array}$ & $\begin{array}{c}\text { Median (Min.- } \\
\text { max.) }\end{array}$ & $\mathbf{p}^{*}$ \\
\hline $\mathrm{A}$ & $\mathrm{n}=30$ & $0.97 \pm 0.09^{\mathrm{a}}$ & $0.99(0.85-1.13)$ & 0.033 \\
$\mathrm{~B}$ & $\mathrm{n}=30$ & $0.96 \pm 0.08^{\mathrm{ab}}$ & $0.97(0.81-1.10)$ & 0.033 \\
$\mathrm{C}$ & $\mathrm{n}=30$ & $0.92 \pm 0.09^{\mathrm{b}}$ & $0.92(0,75-1,09)$ & 0.033 \\
\hline
\end{tabular}

${ }^{\star}$ One-way analysis of variance (ANOVA); ${ }^{\text {a-b }}$ There is no difference between the groups with the same letter.

The mean values of Group A did not exhibit a difference according to gender $(\mathrm{p}=0.445)$. In Group A, while the mean value for women was 0.97 , the mean value for men was 0.99 . The mean values obtained in Group B did not exhibit a difference according to gender $(p=0.709)$. In Group B, while the mean value for women was 0.96 , the mean value for men was 0.95 . The mean values obtained in Group $\mathrm{C}$ did not exhibit a difference according to gender ( $p=0.363$ ). In Group $C$, while the mean value for women was 0.91, the mean value for men was 0.94 (Table 2).

\begin{tabular}{|c|c|c|c|}
\hline & Mean \pm S. deviation & Median (Min.-Max.) & $\mathbf{p}^{*}$ \\
\hline Group A & & & 0.445 \\
\hline Female & $0.97 \pm 0.09$ & $0.97(0.85-1.13)$ & \\
\hline Male & $0.99 \pm 0.09$ & $1.01(0.85-1.1)$ & \\
\hline Group B & & & 0.709 \\
\hline Female & $0.96 \pm 0.08$ & $0.97(0.82-1.1)$ & \\
\hline Male & $0.95 \pm 0.09$ & $0.98(0.81-1.07)$ & \\
\hline Group C & & & 0.363 \\
\hline Female & $0.91 \pm 0.09$ & $0,92(0,75-1,09)$ & \\
\hline Male & $0.94 \pm 0.09$ & $0.93(0.75-1.07)$ & \\
\hline
\end{tabular}

\section{DISCUSSION}

In our study, we performed the fractal dimension analysis on the orthopantomographs to assess the aftermath of the xenograft material placed in the patients who were treated with open sinus lift surgery, focusing on the period of three to six months from the surgery, on the period of six to nine months from the surgery, and on the period of more than nine months from the surgery. The results obtained in the study verify the hypothesis of the study. Although there was no difference between Group B and Groups A, C, we observed that trabeculation increased as the duration increased.

The histological and histomorphometric techniques can be used to examine the mineral quality of the grafted bone and the structure of the trabecular bone, but the invasive nature of the procedure is disadvantageous (16). In the evaluation of hard tissue and hard tissue grafts, Micro-CT is precise and non-invasive. However, the high amount of $\mathrm{x}$-rays projected onto the patient and its cost are the disadvantages of this method (17). Orthopantomographs are widely used to assess the condition of the grafted bone before dental implants are made. While the non-invasive nature of this X-ray-based technique is an advantage, its capability of providing only low-resolution, two-dimensional images is a disadvantage (18). Some properties of orthopantomographs, such as low radiation emission, low cost, and non-invasiveness, are advantageous for postoperative evaluations.

There are various procedures to gain bone height, and open sinus lift surgery has become a standard procedure to enable the placement of dental implants, especially in the presence of insufficient bone in the posterior maxilla (19). Different graft materials such as autografts, xenografts, alloplastic materials, and the combinations of these materials can be used for this procedure (20, 21). Considering the disadvantages of autogenous bone grafts, non-autogenous graft materials are considered an alternative (22). Xenografts are similar to human cancellous bones in terms of their crystalline and morphological structure (23).

Many studies have been carried out to evaluate bones in terms of their quality $(24,25)$. Most of these studies have used the data related to the mineral density measurement of the bone performed with the Dual-energy X-ray absorptiometry method and the data related to the radiomorphometric measurements called panoramic mandibular index (26). These studies evaluating the density and macrostructure of the bone do not provide sufficient information about the trabecular structure of the bone. However, the trabecular bone plays an important role in the durability of the bone, and together with the cortical bone, it determines the biomechanical properties of the bone (27). In recent years, fractal analysis has gained popularity in terms of the detection of possible abnormalities, the evaluation of existing disorders in bone structure, and the evaluation of changes in the bone structure (28). While a high FD value indicates a more complex and dense bone structure, a low FD value indicates a more porous bone structure (29). Measuring and calculating FD is easy, and the measurement area is determined subjectively. The obtained quantitative values provide objective information regarding the tissue of the bone or regarding the bone-like structure. Several studies have evaluated FD in different ways (28-30). The most frequently used method in the literature to calculate FD is the box-counting technique, which was also used in this study (8).

In their study performed on lab rats, Gomes et al. (31) applied xenograft to the socket after tooth extraction, then sacrificed the animals on the 1st, 7th, 14th, 21st, and 49th days, took radiography, and performed fractal analysis. In the grouping made according to duration, 
they did not find any differences between the groups. However, it must be noted that waiting durations after the xenograft application were low and the time differences between the groups were also low in their study. When we check the mean fractal value, it was below 1, which is similar to our study. By using fractal analysis, Dursun et al. (17) compared the xenograft types in patients whom they treated with bilateral two-staged open sinus lift, and found no difference between the two groups. In their study, unlike ours, they took tissue samples from the relevant area six months after the first procedure, scanned these samples with micro ct, and conducted fractal analysis on the images. As a result, they found no difference between the two groups. Their fractal values after six months were above two, and these results are consistent with the results of neither our study nor similar studies (31-33).

\section{CONCLUSION}

In our study, we observed that the density of the xenograft material used in the open sinus lift tended to decrease starting from the period of three to six months after the surgery (Figure). We observed that nine months after the surgery, this density loss increased even more. This indicates that the initially dense areas within the graft resorb over time and are replaced by the trabecular bone. In conclusion, fractal dimension can be used as a method in the evaluation of ossification occurring after open sinus lift surgery

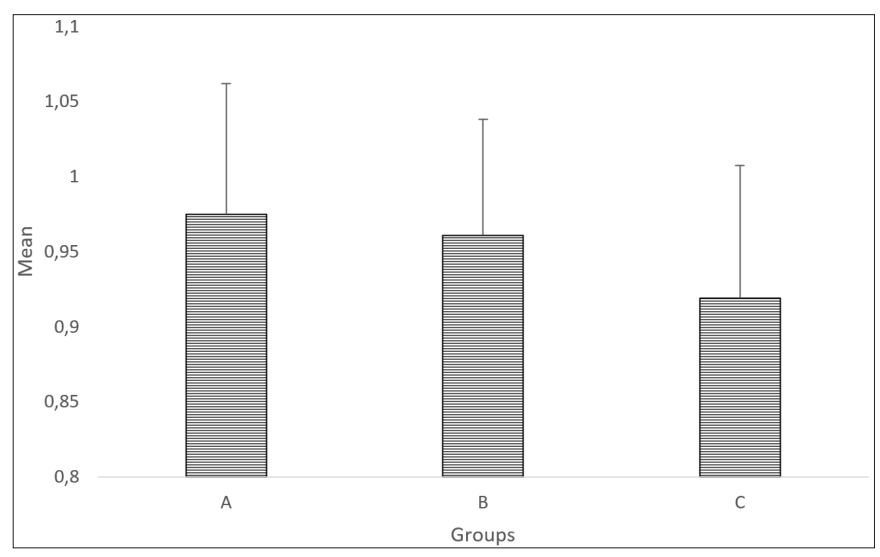

Figure. Mean and standard deviation graph according to groups

\section{ETHICAL DECLARATIONS}

Ethics Committee Approval: Approval for this study was granted by the Health Sciences Non-Invasive Clinical Researchs Ethics Committee of İnönü University (Date: 16-11-2021, Decision No: 2021/2686).

Informed Consent: Because the study was designed retrospectively, no written informed consent form was obtained from patients
Referee Evaluation Process: Externally peer-reviewed. Conflict of Interest Statement: The authors have no conflicts of interest to declare.

Financial Disclosure: The authors declared that this study has received no financial support.

Author Contributions: All of the authors declare that they have all participated in the design, execution, and analysis of the paper and that they have approved the final version.

\section{REFERENCES}

1. Jurisic M, Markovic A, Radulovic M, Brkovic BM, Sándor GK. Maxillary sinus floor augmentation: comparing osteotome with lateral window immediate and delayed implant placements. An interim report. Oral Surg Oral Med Oral Pathol Oral Radiol 2008; 106: 820-7.

2. Albrektsson T, Zarb G, Worthington P, Eriksson A. The long-term efficacy of currently used dental implants: a review and proposed criteria of success. Int J Oral Maxillofac Implants 1986; 1: 11-25.

3. Galindo-Moreno P, Moreno-Riestra I, Ávila G. et al. Histomorphometric comparison of maxillary pristine bone and composite bone graft biopsies obtained after sinus augmentation. Clin Oral Impl Res 2010; 21: 122-8.

4. Aparicio C, Perales P, Rangert B. Tilted implants as an alternative to maxillary sinus grafting: a clinical, radiologic, and periotest study. Clin Implant Dent Relat Res 2001; 3: 39-49.

5. Wallace SS, Froum SJ. Effect of maxillary sinus augmentation on the survival of endosseous dental implants. A systematic review. Ann Periodontol 2003; 8: 328-43.

6. Schlegel KA, Fichtner G, Schultze-Mosgau S, Wiltfang J. Histologic findings in sinus augmentation with autogenous bone chips versus a bovine bone substitute. Int J Oral Maxillofac Implants 2003; 18.

7. Sanchez-Molina D, Velazquez-Ameijide J, Quintana V. et al. Fractal dimension and mechanical properties of human cortical bone. Med Eng \& Phys 2013; 35: 576-82.

8. Sánchez I, Uzcátegui G. Fractals in dentistry. J Dent 2011; 39: 27392.

9. Perrier E, Bird N, Rieu M. Generalizing the fractal model of soil structure: the pore-solid fractal approach. Dev in Soil Science 2000; 47-74.

10. Saeed SS, Ibraheem UM, Alnema MM. Quantitative analysis by pixel intensity and fractal dimensions for imaging diagnosis of periapical lesions. Int J Enhanc Res Sci Technol Eng 2014; 3: 13844.

11. Chen S-K, Oviir T, Lin C-H, Leu L-J, Cho B-H, Hollender L. Digital imaging analysis with mathematical morphology and fractal dimension for evaluation of periapical lesions following endodontic treatment. OOOOE 2005; 100: 467-72.

12.Bollen AM, Taguchi A, Hujoel PP, Hollender L. G. . Fractal dimension on dental radiographs. Dentomaxillofac Radiol 2001; 30: 270-5.

13. Baig MA, Bacha D. Histology, Bone. StatPearls [Internet] 2021.

14. Rosen CJ. Pathogenesis of osteoporosis. Best Pract Res Clin Endocrinol Metab 2000; 14: 181-93.

15. White SC, Rudolph DJ. Alterations of the trabecular pattern of the jaws in patients with osteoporosis. Oral Surg Oral Med Oral Path Oral Radio Endod 1999; 88: 628-35.

16.Gedrange T, Hietschold V, Mai R, Wolf P, Nicklisch M, Harzer W. An evaluation of resonance frequency analysis for the determination of the primary stability of orthodontic palatal implants. A study in human cadavers. Clin Oral Impl Res 2005; 16: $425-31$. 
17. Dursun E, Dursun CK, Eratalay K, Orhan K, Celik HH, Tözüm TF. Do porous titanium granule grafts affect bone microarchitecture at augmented maxillary sinus sites? A pilot split-mouth human study. Implant Dent 2015; 24: 427-33.

18. Huang HL, Chen MY, Hsu JT, Li YF, Chang CH, Chen KT. Three-dimensional bone structure and bone mineral density evaluations of autogenous bone graft after sinus augmentation: a microcomputed tomography analysis. Clin Oral Impl Res 2012; 23: 1098-103.

19. Browaeys H, Defrancq J, Dierens MC, et al. A retrospective analysis of early and immediately loaded osseotite implants in cross-arch rehabilitations in edentulous maxillas and mandibles up to 7 years. Clin Implant Dent Relat Res 2013; 15: 380-9.

20. Ramírez-Fernández MP, Calvo-Guirado JL, Maté-Sánchez del Val JE, Delgado-Ruiz RA, Negri B, Barona-Dorado C. Ultrastructural study by backscattered electron imaging and elemental microanalysis of bone-to-biomaterial interface and mineral degradation of porcine xenografts used in maxillary sinus floor elevation. Clin Oral Impl Res 2013; 24: 523-30.

21. Orsini G, Scarano A, Piattelli M, Piccirilli M, Caputi S, Piattelli A. Histologic and ultrastructural analysis of regenerated bone in maxillary sinus augmentation using a porcine bone-derived biomaterial. J Periodontol 2006; 77: 1984-90.

22. Al-Nawas B, Schiegnitz E. Augmentation procedures using bone substitute materials or autogenous bone-a systematic review and meta-analysis. Eur J Oral Implantol 2014; 7: 219-34.

23. Yildirim M, Spiekermann H, Biesterfeld S, Edelhoff D. Maxillary sinus augmentation using xenogenic bone substitute material Bio-Oss ${ }^{\infty}$ in combination with venous blood: A histologic and histomorphometric study in humans. Clin Oral Impl Res 2000; 11: 217-29.

24. Fazzalari N, Parkinson I. Fractal properties of cancellous bone of the iliac crest in vertebral crush fracture. Bone 1998; 23: 53-7.

25. Cakur B, Şahin A, Dagistan S, et al. Dental panoramic radiography in the diagnosis of osteoporosis. J Int Med Res 2008; 36: 792-9.

26.Dagistan S, Bilge O. Comparison of antegonial index, mental index, panoramic mandibular index and mandibular cortical index values in the panoramic radiographs of normal males and male patients with osteoporosis. Dentomaxillofac Radiol 2010; 39: $290-4$.

27.Carballido-Gamio J, Majumdar S. Clinical utility of microarchitecture measurements of trabecular bone. Curr Osteoporos Reports 2006; 4: 64-70.

28.Demiralp KÖ, Kurșun-Çakmak EŞ, Bayrak S, Akbulut N, Atakan C, Orhan K. Trabecular structure designation using fractal analysis technique on panoramic radiographs of patients with bisphosphonate intake: a preliminary study. Oral Radiol 2019; 35: 23-8.

29. Arsan B, Köse TE, Cene E, Özcan İ. Assessment of the trabecular structure of mandibular condyles in patients with temporomandibular disorders using fractal analysis. Oral Surg Oral Med Oral Pathol Oral Radiol 2017; 123: 382-91.

30.Göller Bulut D, Bayrak S, Uyeturk U, Ankarali H. Mandibular indexes and fractal properties on the panoramic radiographs of the patients using aromatase inhibitors. Br J Radiol 2018; 91: 20180442.

31. Gomes NR, Albergaria JD, Henriques JAdS, et al. Comparison between fractal analysis and radiopacity evaluation as a tool for studying repair of an osseous defect in an animal model using biomaterials. Dentomaxillofacial Radiol 2019; 48: 20180466.

32. Ustaoğlu G, Bulut DG, Gümüş K. Evaluation of different plateletrich concentrates effects on early soft tissue healing and socket preservation after tooth extraction. J Stomatol Oral Maxillofac Surg 2020; 121: 539-44.

33. Gümüşsoy İ. Application of fractal analysis method on panoramic radiographs and effect of different X-Ray devices on fractal dimension value. Sakarya Tip Derg 9: 492-8. 